\title{
Learning with Unlabeled Data for Text Categorization Using Bootstrapping and Feature Projection Techniques
}

\author{
Youngjoong Ko \\ Dept. of Computer Science, Sogang Univ. \\ Sinsu-dong 1, Mapo-gu \\ Seoul, 121-742, Korea \\ kyj@nlpzodiac.sogang.ac.kr
}

\author{
Jungyun Seo \\ Dept. of Computer Science, Sogang Univ. \\ Sinsu-dong 1, Mapo-gu \\ Seoul, 121-742, Korea \\ seojy@ccs.sogang.ac.kr
}

\begin{abstract}
A wide range of supervised learning algorithms has been applied to Text Categorization. However, the supervised learning approaches have some problems. One of them is that they require a large, often prohibitive, number of labeled training documents for accurate learning. Generally, acquiring class labels for training data is costly, while gathering a large quantity of unlabeled data is cheap. We here propose a new automatic text categorization method for learning from only unlabeled data using a bootstrapping framework and a feature projection technique. From results of our experiments, our method showed reasonably comparable performance compared with a supervised method. If our method is used in a text categorization task, building text categorization systems will become significantly faster and less expensive.
\end{abstract}

\section{Introduction}

Text categorization is the task of classifying documents into a certain number of pre-defined categories. Many supervised learning algorithms have been applied to this area. These algorithms today are reasonably successful when provided with enough labeled or annotated training examples. For example, there are Naive Bayes (McCallum and Nigam, 1998), Rocchio (Lewis et al., 1996), Nearest Neighbor $(k N N)$ (Yang et al., 2002), TCFP (Ko and Seo, 2002), and Support Vector Machine (SVM) (Joachims, 1998).

However, the supervised learning approach has some difficulties. One key difficulty is that it requires a large, often prohibitive, number of labeled training data for accurate learning. Since a labeling task must be done manually, it is a painfully time-consuming process. Furthermore, since the application area of text categorization has diversified from newswire articles and web pages to E-mails and newsgroup postings, it is also a difficult task to create training data for each application area (Nigam et al., 1998). In this light, we consider learning algorithms that do not require such a large amount of labeled data.

While labeled data are difficult to obtain, unlabeled data are readily available and plentiful. Therefore, this paper advocates using a bootstrapping framework and a feature projection technique with just unlabeled data for text categorization. The input to the bootstrapping process is a large amount of unlabeled data and a small amount of seed information to tell the learner about the specific task. In this paper, we consider seed information in the form of title words associated with categories. In general, since unlabeled data are much less expensive and easier to collect than labeled data, our method is useful for text categorization tasks including online data sources such as web pages, E-mails, and newsgroup postings.

To automatically build up a text classifier with unlabeled data, we must solve two problems; how we can automatically generate labeled training documents (machine-labeled data) from only title words and how we can handle incorrectly labeled documents in the machine-labeled data. This paper provides solutions for these problems. For the first problem, we employ the bootstrapping framework. For the second, we use the TCFP classifier with robustness from noisy data (Ko and Seo, 2004).

How can labeled training data be automatically created from unlabeled data and title words? Maybe unlabeled data don't have any information for building a text classifier because they do not contain the most important information, their category. Thus we must assign the class to each document in order to use supervised learning approaches. Since text categorization is a task based on pre-defined categories, we know the categories for classifying documents. Knowing the categories means that we can choose at least a representative title word of each category. This is the starting point of our proposed method. As we carry out a bootstrapping task from these title words, we can finally get labeled training data. Suppose, for example, that we are interested in classifying newsgroup postings about specially 
'Autos' category. Above all, we can select 'automobile' as a title word, and automatically extract keywords ('car', 'gear', 'transmission', 'sedan', and so on) using co-occurrence information. In our method, we use context ( $a$ sequence of 60 words) as a unit of meaning for bootstrapping from title words; it is generally constructed as a middle size of a sentence and a document. We then extract core contexts that include at least one of the title words and the keywords. We call them centroid-contexts because they are regarded as contexts with the core meaning of each category. From the centroidcontexts, we can gain many words contextually cooccurred with the title words and keywords: 'driver', 'clutch', 'trunk', and so on. They are words in first-order co-occurrence with the title words and the keywords. To gather more vocabulary, we extract contexts that are similar to centroid-contexts by a similarity measure; they contain words in second-order co-occurrence with the title words and the keywords. We finally construct context-cluster of each category as the combination of centroid-contexts and contexts selected by the similarity measure. Using the context-clusters as labeled training data, a Naive Bayes classifier can be built. Since the Naive Bayes classifier can label all unlabeled documents for their category, we can finally obtain labeled training data (machine-labeled data).

When the machine-labeled data is used to learn a text classifier, there is another difficult in that they have more incorrectly labeled documents than manually labeled data. Thus we develop and employ the TCFP classifiers with robustness from noisy data.

The rest of this paper is organized as follows. Section 2 reviews previous works. In section 3 and 4 , we explain the proposed method in detail. Section 5 is devoted to the analysis of the empirical results. The final section describes conclusions and future works.

\section{Related Works}

In general, related approaches for using unlabeled data in text categorization have two directions; One builds classifiers from a combination of labeled and unlabeled data (Nigam, 2001; Bennett and Demiriz, 1999), and the other employs clustering algorithms for text categorization (Slonim et al., 2002).

Nigam studied an Expected Maximization (EM) technique for combining labeled and unlabeled data for text categorization in his dissertation. $\mathrm{He}$ showed that the accuracy of learned text classifiers can be improved by augmenting a small number of labeled training data with a large pool of unlabeled data.

Bennet and Demiriz achieved small improvements on some UCI data sets using SVM. It seems that SVMs assume that decision boundaries lie between classes in low-density regions of instance space, and the unlabeled examples help find these areas.

Slonim suggested clustering techniques for unsupervised document classification. Given a collection of unlabeled data, he attempted to find clusters that are highly correlated with the true topics of documents by unsupervised clustering methods. In his paper, Slonim proposed a new clustering method, the sequential Information Bottleneck (sIB) algorithm.

\section{The Bootstrapping Algorithm for Creating Machine-labeled Data}

The bootstrapping framework described in this paper consists of the following steps. Each module is described in the following sections in detail.

1. Preprocessing: Contexts are separated from unlabeled documents and content words are extracted from them.

2. Constructing context-clusters for training:

- Keywords of each category are created

- Centroid-contexts are extracted and verified

- Context-clusters are created by a similarity measure

3. Learning Classifier: Naive Bayes classifier are learned by using the context-clusters

\subsection{Preprocessing}

The preprocessing module has two main roles: extracting content words and reconstructing the collected documents into contexts. We use the Brill POS tagger to extract content words (Brill, 1995).

Generally, the supervised learning approach with labeled data regards a document as a unit of meaning. But since we can use only the title words and unlabeled data, we define context as a unit of meaning and we employ it as the meaning unit to bootstrap the meaning of each category. In our system, we regard a sequence of 60 content words within a document as a context. To extract contexts from a document, we use sliding window techniques (Maarek et al., 1991). The window is a slide from the first word of the document to the last in the size of the window (60 words) and the interval of each window (30 words). Therefore, the final output of preprocessing is a set of context vectors that are represented as content words of each context. 


\subsection{Constructing Context-Clusters for Training}

At first, we automatically create keywords from a title word for each category using co-occurrence information. Then centroid-contexts are extracted using the title word and keywords. They contain at least one of the title and keywords. Finally, we can gain more information of each category by assigning remaining contexts to each contextcluster using a similarity measure technique; the remaining contexts do not contain any keywords or title words.

\subsubsection{Creating Keyword Lists}

The starting point of our method is that we have title words and collected documents. A title word can present the main meaning of each category but it could be insufficient in representing any category for text categorization. Thus we need to find words that are semantically related to a title word, and we define them as keywords of each category.

The score of semantic similarity between a title word, $T$, and a word, $W$, is calculated by the cosine metric as follows:

$$
\operatorname{sim}(T, W)=\frac{\sum_{i=1}^{n} t_{i} \times w_{i}}{\sqrt{\sum_{i=1}^{n} t_{i}^{2} \times \sum_{i=1}^{n} w_{i}^{2}}}
$$

where $t_{i}$ and $w_{i}$ represent the occurrence (binary value: 0 or 1 ) of words $T$ and $W$ in $i$-th document respectively, and $n$ is the total number of documents in the collected documents. This method calculates the similarity score between words based on the degree of their co-occurrence in the same document.

Since the keywords for text categorization must have the power to discriminate categories as well as similarity with the title words, we assign a word to the keyword list of a category with the maximum similarity score and recalculate the score of the word in the category using the following formula:

$$
\operatorname{Score}\left(W, c_{\max }\right)=\operatorname{sim}\left(T_{\max }, W\right)+\left(\operatorname{sim}\left(T_{\max }, W\right)-\operatorname{sim}\left(T_{\text {second } \max }, W\right)\right)
$$

where $T_{\max }$ is the title word with the maximum similarity score with a word $W, c_{\max }$ is the category of the title word $T_{\max }$, and $T_{\text {secondmax }}$ is other title word with the second high similarity score with the word $W$.

This formula means that a word with high ranking in a category has a high similarity score with the title word of the category and a high similarity score difference with other title words.
We sort out words assigned to each category according to the calculated score in descending order. We then choose top $m$ words as keywords in the category. Table 1 shows the list of keywords (top 5) for each category in the WebKB data set.

Table 1. The list of keywords in the WebKB data set

\begin{tabular}{|c||c|c|}
\hline Category & Title Word & Keywords \\
\hline \hline course & course & $\begin{array}{c}\text { assignments, hours, instructor, } \\
\text { class, fall }\end{array}$ \\
\hline faculty & professor & $\begin{array}{c}\text { associate, ph.d, fax, interests, } \\
\text { publications }\end{array}$ \\
\hline project & project & $\begin{array}{c}\text { system, systems, research, } \\
\text { software, information }\end{array}$ \\
\hline student & student & $\begin{array}{c}\text { graduate, computer, science, } \\
\text { page, university }\end{array}$ \\
\hline
\end{tabular}

\subsubsection{Extracting and Verifying Centroid-Contexts}

We choose contexts with a keyword or a title word of a category as centroid-contexts. Among centroid-contexts, some contexts could not have good features of a category even though they include the keywords of the category. To rank the importance of centroid-contexts, we compute the importance score of each centroid-context. First of all, weights $\left(W_{i j}\right)$ of word $w_{i}$ in $j$-th category are calculated using Term Frequency $(T F)$ within a category and Inverse Category Frequency (ICF) (Cho and Kim, 1997) as follows:

$$
W_{i j}=T F_{i j} \times I C F_{i}=T F_{i j} \times\left(\log (M)-\log \left(C F_{i}\right)\right)
$$

where $C F_{i}$ is the number of categories that contain $w_{i}$ and $M$ is the total number of categories.

Using word weights $\left(W_{i j}\right)$ calculated by formula 3 , the score of a centroid-context $\left(S_{k}\right)$ in $j$-th category $\left(c_{j}\right)$ is computed as follows:

$$
\operatorname{Score}\left(S_{k}, c_{j}\right)=\frac{W_{1 j}+W_{2 j}+\ldots+W_{N j}}{N}
$$

where $\mathrm{N}$ is the number of words in the centroidcontext.

As a result, we obtain a set of words in firstorder co-occurrence from centroid-contexts of each category.

\subsubsection{Creating Context-Clusters}

We gather the second-order co-occurrence information by assigning remaining contexts to the context-cluster of each category. For the assigning criterion, we calculate similarity between remaining contexts and centroid-contexts of each category. Thus we employ the similarity measure technique by Karov and Edelman (1998). In our method, a part of this technique is reformed for our 
purpose and remaining contexts are assigned to each context-cluster by that revised technique.

\section{1) Measurement of word and context similarities}

As similar words tend to appear in similar contexts, we can compute the similarity by using contextual information. Words and contexts play complementary roles. Contexts are similar to the extent that they contain similar words, and words are similar to the extent that they appear in similar contexts (Karov and Edelman, 1998). This definition is circular. Thus it is applied iteratively using two matrices, WSM and CSM.

Each category has a word similarity matrix $W S M_{\mathrm{n}}$ and a context similarity matrix $C S M_{\mathrm{n}}$. In each iteration $n$, we update $W S M_{n}$, whose rows and columns are labeled by all content words encountered in the centroid-contexts of each category and input remaining contexts. In that matrix, the cell $(i, j)$ holds a value between 0 and 1 , indicating the extent to which the $i$-th word is contextually similar to the $j$-th word. Also, we keep and update a $\mathrm{CSM}_{n}$, which holds similarities among contexts. The rows of $\mathrm{CSM}_{n}$ correspond to the remaining contexts and the columns to the centroid-contexts. In this paper, the number of input contexts of row and column in CSM is limited to 200, considering execution time and memory allocation, and the number of iterations is set as 3 .

To compute the similarities, we initialize $W S M_{n}$ to the identity matrix. The following steps are iterated until the changes in the similarity values are small enough.

1. Update the context similarity matrix $\mathrm{CSM}_{n}$, using the word similarity matrix $W S M_{n}$.

2. Update the word similarity matrix $W S M_{n}$, using the context similarity matrix $\mathrm{CSM}_{n}$.

\section{2) Affinity formulae}

To simplify the symmetric iterative treatment of similarity between words and contexts, we define an auxiliary relation between words and contexts as affinity.

Affinity formulae are defined as follows (Karov and Edelman, 1998):

$$
\begin{aligned}
& \operatorname{aff}_{n}(W, X)=\max _{W_{i} \in X} \operatorname{sim}_{n}\left(W, W_{i}\right) \\
& \operatorname{aff}_{n}(X, W)=\max _{W \in X_{j}} \operatorname{sim}_{n}\left(X, X_{j}\right)
\end{aligned}
$$

In the above formulae, $n$ denotes the iteration number, and the similarity values are defined by $W S M_{n}$ and $C S M_{n}$. Every word has some affinity to the context, and the context can be represented by a vector indicating the affinity of each word to it.

\section{3) Similarity formulae}

The similarity of $W_{1}$ to $W_{2}$ is the average affinity of the contexts that include $W_{1}$ to $W_{2}$, and the similarity of a context $X_{1}$ to $X_{2}$ is a weighted average of the affinity of the words in $X_{1}$ to $X_{2}$. Similarity formulae are defined as follows:

$$
\begin{aligned}
& \operatorname{sim}_{n+1}\left(X_{1}, X_{2}\right)=\sum_{W \in X_{1}} \text { weight }\left(W, X_{1}\right) \cdot \operatorname{aff}_{n}\left(W, X_{2}\right) \\
& \text { if } W_{1}=W_{2} \\
& \operatorname{sim}_{n+1}\left(W_{1}, W_{2}\right)=1 \\
& \text { else } \\
& \operatorname{sim}_{n+1}\left(W_{1}, W_{2}\right)=\sum_{W_{1} \in X} \operatorname{weight}\left(X, W_{1}\right) \cdot \operatorname{aff}_{n}\left(X, W_{2}\right)
\end{aligned}
$$

The weights in formula 7 are computed as reflecting global frequency, log-likelihood factors, and part of speech as used in (Karov and Edelman, 1998). The sum of weights in formula 8, which is a reciprocal number of contexts that contain $W_{l}$, is 1 .

\section{4) Assigning remaining contexts to a category}

We decided a similarity value of each remaining context for each category using the following method:

$$
\operatorname{sim}_{c_{i} \in C}\left(X, c_{i}\right)=\operatorname{aver}\left\{\operatorname{sim}_{S_{j} \in C C_{c_{i}}}\left(X, S_{j}\right)\right\}
$$

In formula 9, i) $X$ is a remaining context, ii) $C=\left\{c_{1}, c_{2}, \ldots, c_{m}\right\}$ is a category set, and iii) $C C_{c_{i}}=\left\{S_{1}, \ldots, S_{n}\right\}$ is a controid-contexts set of category $c_{i}$.

Each remaining context is assigned to a category which has a maximum similarity value. But there may exist noisy remaining contexts which do not belong to any category. To remove these noisy remaining contexts, we set up a dropping threshold using normal distribution of similarity values as follows (Ko and Seo, 2000):

$$
\max \left\{\operatorname{sim}_{\mathrm{c}_{\mathrm{i}} \in \mathrm{C}}\left(X, c_{i}\right)\right\} \geq \mu+\theta \sigma
$$

where i) $X$ is a remaining context, ii) $\mu$ is an average of similarity values $\operatorname{sim}_{c_{i} \in C}\left(X, c_{i}\right)$, iii) $\sigma$ is a standard deviation of similarity values, and iv) $\theta$ is a numerical value corresponding to the threshold (\%) in normal distribution table.

Finally, a remaining context is assigned to the context-cluster of any category when the category has a maximum similarity above the dropping threshold value. In this paper, we empirically use a $15 \%$ threshold value from an experiment using a validation set. 


\subsection{Learning the Naive Bayes Classifier Using Context-Clusters}

In above section, we obtained labeled training data: context-clusters. Since training data are labeled as the context unit, we employ a Naive Bayes classifier because it can be built by estimating the word probability in a category, but not in a document. That is, the Naive Bayes classifier does not require labeled data with the unit of documents unlike other classifiers.

We use the Naive Bayes classifier with minor modifications based on Kullback-Leibler Divergence (Craven et al., 2000). We classify a document $d_{i}$ according to the following formula:

$$
\begin{aligned}
P\left(c_{j} \mid d_{i} ; \hat{\theta}\right) & =\frac{P\left(c_{j} \mid \hat{\theta}\right) P\left(d_{i} \mid c_{j} ; \hat{\theta}\right)}{P\left(d_{i} \mid \hat{\theta}\right)} \approx P\left(c_{j} \mid \hat{\theta} \prod_{t=1}^{|W|} P\left(w_{t} \mid c_{j} ; \hat{\theta}^{N\left(w, d_{i}\right)}\right.\right. \\
& \propto \frac{\log P\left(c_{j} ; \hat{\theta}\right)}{n}+\sum_{t=1}^{|W|} P\left(w_{t} \mid d_{i} ; \hat{\theta}\right) \log \left(\frac{P\left(w_{t} \mid c_{j} ; \hat{\theta}\right)}{P\left(w_{t} \mid d_{i} ; \hat{\theta}\right)}\right)
\end{aligned}
$$

where i) $n$ is the number of words in document $d_{i}$, ii) $w_{t}$ is the $t$-th word in the vocabulary, iii) $N\left(w_{t}, d_{i}\right)$ is the frequency of word $w_{t}$ in document $d_{i}$.

Here, the Laplace smoothing is used to estimate the probability of word $w_{t}$ in class $c_{j}$ and the probability of class $c_{j}$ as follows:

$$
\begin{gathered}
P\left(w_{t} \mid c_{j} ; \hat{\theta}\right)=\frac{1+N\left(w_{t}, G_{c_{j}}\right)}{|V|+\sum_{t=1}^{|V|} N\left(w_{t}, G_{c_{j}}\right)} \\
P\left(c_{j} \mid \hat{\theta}\right)=\frac{1+\left|G_{c_{j}}\right|}{|C|+\sum_{c_{i}}\left|G_{c_{i}}\right|}
\end{gathered}
$$

where $N\left(w_{t}, G_{c_{j}}\right)$ is the count of the number of times word $w_{\mathrm{t}}$ occurs in the context-cluster $\left(G_{c_{j}}\right)$ of category $c_{j}$.

\section{Using a Feature Projection Technique for Handling Noisy Data of Machine-labeled Data}

We finally obtained labeled data of a documents unit, machine-labeled data. Now we can learn text classifiers using them. But since the machinelabeled data are created by our method, they generally include far more incorrectly labeled documents than the human-labeled data. Thus we employ a feature projection technique for our method. By the property of the feature projection technique, a classifier (the TCFP classifier) can have robustness from noisy data (Ko and Seo, 2004). As seen in our experiment results, TCFP showed the highest performance among conventional classifiers in using machine-labeled data.

\section{The TCFP classifier with robustness from noisy data}

Here, we simply describe the TCFP classifier using the feature projection technique (Ko and Seo, 2002 ; 2004). In this approach, the classification knowledge is represented as sets of projections of training data on each feature dimension. The classification of a test document is based on the voting of each feature of that test document. That is, the final prediction score is calculated by accumulating the voting scores of all features.

First of all, we must calculate the voting ratio of each category for all features. Since elements with a high TF-IDF value in projections of a feature must become more useful classification criteria for the feature, we use only elements with TF-IDF values above the average TF-IDF value for voting. And the selected elements participate in proportional voting with the same importance as the TF-IDF value of each element. The voting ratio of each category $c_{j}$ in a feature $t_{m}$ is calculated by the following formula:

$$
r\left(c_{j}, t_{m}\right)=\sum_{t_{m}(l) \in I_{m}} w\left(t_{m}, \vec{d}_{l}\right) \cdot y\left(c_{j}, t_{m}(l)\right) / \sum_{t_{m}(l) \in I_{m}} w\left(t_{m}, \vec{d}_{l}\right)
$$

In formula $14, w\left(t_{m}, \vec{d}\right)$ is the weight of term $t_{m}$ in document $d, I_{m}$ denotes a set of elements selected for voting and $y\left(c_{j}, t_{m}(l)\right) \in\{0.1\}$ is a function; if the category for an element $t_{m}(l)$ is equal to $c_{j}$, the output value is 1 . Otherwise, the output value is 0 .

Next, since each feature separately votes on feature projections, contextual information is missing. Thus we calculate co-occurrence frequency of features in the training data and modify TF-IDF values of two terms $t_{i}$ and $t_{j}$ in a test document by co-occurrence frequency between them; terms with a high co-occurrence frequency value have higher term weights.

Finally, the voting score of each category $c_{j}$ in the $m$-th feature $t_{m}$ of a test document $d$ is calculated by the following formula:

$$
v s\left(c_{j}, t_{m}\right)=t w\left(t_{m}, \vec{d}\right) \cdot r\left(c_{j}, t_{m}\right) \cdot \log \left(1+\chi^{2}\left(t_{m}\right)\right)
$$

where $t w\left(t_{m}, d\right)$ denotes a modified term weight by the co-occurrence frequency and $\chi^{2}\left(t_{m}\right)$ denotes the calculated $\chi^{2}$ statistics value of $t_{m}$. 
Table 2. The top micro-avg F1 scores and precision-recall breakeven points of each method.

\begin{tabular}{|l||c|c|c|c|c|c|}
\hline & $\begin{array}{c}\text { OurMethod } \\
(\text { basis })\end{array}$ & $\begin{array}{c}\text { OurMethod } \\
(\text { NB })\end{array}$ & $\begin{array}{c}\text { OurMethod } \\
(\text { Rochhio })\end{array}$ & $\begin{array}{c}\text { OurMethod } \\
(\text { kNN) }\end{array}$ & $\begin{array}{c}\text { OurMethod } \\
(\text { SVM })\end{array}$ & $\begin{array}{c}\text { OurMethod } \\
(\text { TCFP })\end{array}$ \\
\hline \hline Newsgroups & $\mathbf{7 9 . 3 6}$ & 83.46 & 83 & 79.95 & 82.49 & $\mathbf{8 6 . 1 9}$ \\
\hline WebKB & $\mathbf{7 3 . 6 3}$ & 73.22 & 75.28 & 68.04 & 73.74 & 75.47 \\
\hline Reuters & $\mathbf{8 8 . 6 2}$ & 88.23 & 86.26 & 85.65 & 87.41 & $\mathbf{8 9 . 0 9}$ \\
\hline
\end{tabular}

The outline of the TCFP classifier is as follow:

1. input : test document: $\vec{d}=\left\langle t_{1}, t_{2}, \ldots, t_{n}\right\rangle$

2. main process

For each feature $t_{i}$ $t w\left(t_{i}, d\right)$ is calculated

For each feature $t_{i}$

For each category $c_{j}$ $\operatorname{vote}\left[c_{j}\right]=\operatorname{vote}\left[c_{j}\right]+v s\left(c_{j}, t_{i}\right)$ by Formula 15

prediction $=\arg \max \operatorname{vote}\left[c_{j}\right]$

\section{Empirical Evaluation}

\subsection{Data Sets and Experimental Settings}

To test our method, we used three different kinds of data sets: UseNet newsgroups (20 Newsgroups), web pages (WebKB), and newswire articles (Reuters 21578). For fair evaluation in Newsgroups and WebKB, we employed the fivefold cross-validation method.

The Newsgroups data set, collected by Ken Lang, contains about 20,000 articles evenly divided among 20 UseNet discussion groups (McCallum and Nigam, 1998). In this paper, we used only 16 categories after removing 4 categories: three miscellaneous categories (talk.politics.misc, talk.religion.misc, and comp.os.ms-windows.misc) and one duplicate meaning category (comp.sys. ibm.pc.hardware).

The second data set comes from the WebKB project at CMU (Craven et al., 2000). This data set contains web pages gathered from university computer science departments.

The Reuters 21578 Distribution 1.0 data set consists of 12,902 articles and 90 topic categories from the Reuters newswire. Like other study in (Nigam, 2001), we used the ten most populous categories to identify the news topic.

About 25\% documents from training data of each data set are selected for a validation set. We applied a statistical feature selection method $\left(\chi^{2}\right.$ statistics) to a preprocessing stage for each classifier (Yang and Pedersen, 1997).

As performance measures, we followed the standard definition of recall, precision, and $F_{1}$ measure. For evaluation performance average across categories, we used the micro-averaging method (Yang et al., 2002). Results on Reuters are reported as precision-recall breakeven points, which is a standard information retrieval measure for binary classification (Joachims, 1998).

Title words in our experiment are selected according to category names of each data set (see Table 1 as an example).

\subsection{Experimental Results}

\subsubsection{Observing the Performance According to the Number of Keywords}

First of all, we determine the number of keywords in our method using the validation set. The number of keywords is limited by the top $m$-th keyword from the ordered list of each category. Figure 1 displays the performance at different number of keywords (from 0 to 20) in each data set.

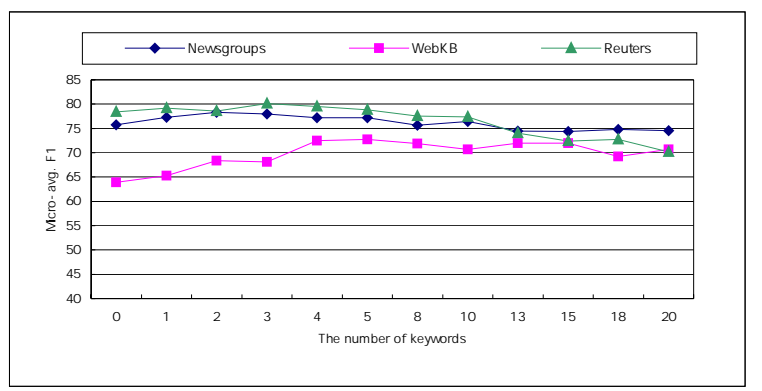

Figure 1. The comparison of performance according to the number of keywords

We set the number of keywords to 2 in Newsgroups, 5 in WebKB, and 3 in Reuters empirically. Generally, we recommend that the number of keywords be between 2 and 5 .

\subsubsection{Comparing our Method Using TCFP with those Using other Classifiers}

In this section, we prove the superiority of TCFP over the other classifiers (SVM, $k N N$, Naive Bayes (NB), Roccio) in training data with much noisy data such as machine-labeled data. As shown in Table 2, we obtained the best performance in using TCFP at all three data sets.

Let us define the notations. OurMethod(basis) denotes the Naive Bayes classifier using labeled contexts and OurMethod(NB) denotes the Naive Bayes classifier using machine-labeled data as 
training data. The same manner is applied for other classifiers.

OurMethod(TCFP) achieved more advanced scores than OurMethod(basis): 6.83 in Newsgroups, 1.84 in WebKB, and 0.47 in Reuters.

\subsubsection{Comparing with the Supervised Naive Bayes Classifier}

For this experiment, we consider two possible cases for labeling task. The first task is to label a part of collected documents and the second is to label all of them. As the first task, we built up a new training data set; it consists of 500 different documents randomly chosen from appropriate categories like the experiment in (Slonim et al., 2002). As a result, we report performances from two kinds of Naive Bayes classifiers which are learned from 500 training documents and the whole training documents respectively.

Table 3. The comparison of our method and the supervised NB classifier

\begin{tabular}{|l||c|c|c|}
\hline & $\begin{array}{c}\text { OurMethod } \\
(\text { TCFP })\end{array}$ & $\begin{array}{c}\text { NB } \\
(\mathbf{5 0 0})\end{array}$ & $\begin{array}{c}\text { NB } \\
(\text { All })\end{array}$ \\
\hline \hline Newsgroups & 86.19 & 72.68 & 91.72 \\
\hline WebKB & 75.47 & 74.1 & 85.29 \\
\hline Reuters & 89.09 & 82.1 & 91.64 \\
\hline
\end{tabular}

In Table 3, the results of our method are higher than those of $N B(500)$ and are comparable to those of $N B(A l l)$ in all data sets. Especially, the result in Reuters reached 2.55 close to that of $N B(A l l)$ though it used the whole labeled training data.

\subsubsection{Enhancing our Method from Choosing Keywords by Human}

The main problem of our method is that the performance depends on the quality of the keywords and title words. As we have seen in Table 3, we obtained the worst performance in the WebKB data set. In fact, title words and keywords of each category in the WebKB data set also have high frequency in other categories. We think these factors contribute to a comparatively poor performance of our method. If keywords as well as title words are supplied by humans, our method may achieve higher performance. However, choosing the proper keywords for each category is a much difficult task. Moreover, keywords from developers, who have insufficient knowledge about an application domain, do not guarantee high performance. In order to overcome this problem, we propose a hybrid method for choosing keywords. That is, a developer obtains 10 candidate keywords from our keyword extraction method and then they can choose proper keywords from them. Table 4 shows the results from three data sets.
Table 4. The comparison of our method and enhancing method

\begin{tabular}{|l||c|c|c|}
\hline & $\begin{array}{c}\text { OurMethod } \\
\text { (TCFP) }\end{array}$ & $\begin{array}{c}\text { Enhancing } \\
\text { (TCFP)) }\end{array}$ & Improvement \\
\hline \hline Newsgroups & 86.19 & 86.23 & $\mathbf{+ 0 . 0 4}$ \\
\hline WebKB & 75.47 & 77.59 & $\mathbf{+ 2 . 1 2}$ \\
\hline Reuters & 89.09 & 89.52 & $\mathbf{+ 0 . 4 3}$ \\
\hline
\end{tabular}

As shown in Table 4, especially we could achieve significant improvement in the WebKb data set. Thus we find that the new method for choosing keywords is more useful in a domain with confused keywords between categories such as the WebKB data set.

\subsubsection{Comparing with a Clustering Technique}

In related works, we presented two approaches using unlabeled data in text categorization; one approach combines unlabeled data and labeled data, and the other approach uses the clustering technique for text categorization. Since our method does not use any labeled data, it cannot be fairly compared with the former approaches. Therefore, we compare our method with a clustering technique. Slonim et al. (2002) proposed a new clustering algorithm (sIB) for unsupervised document classification and verified the superiority of his algorithm. In his experiments, the $\boldsymbol{s I \boldsymbol { B }}$ algorithm was superior to other clustering algorithms. As we set the same experimental settings as in Slonim's experiments and conduct experiments, we verify that our method outperforms ths $\boldsymbol{s} \boldsymbol{I B}$ algorithm. In our experiments, we used the micro-averaging precision as performance measure and two revised data sets: revised_NG, revised_Reuters. These data sets were revised in the same way according to Slonim's paper as follows:

In revised_NG, the categories of Newsgroups were united with respect to 10 meta-categories: five comp categories, three politics categories, two sports categories, three religions categories, and two transportation categories into five big metacategories.

The revised_Reuters used the 10 most frequent categories in the Reuters 21578 corpus under the ModApte split.

As shown in Table 5, our method shows 6.65 advanced score in revised_NG and 3.2 advanced score in revised_Reuters.

Table 5. The comparison of our method and $s I B$

\begin{tabular}{|l||c|c|c|}
\hline & sIB & $\begin{array}{c}\text { OurMethod } \\
(\text { TCFP })\end{array}$ & Improvement \\
\hline \hline revised_NG & 79.5 & 86.15 & $\mathbf{+ 6 . 6 5}$ \\
\hline revised_Reuters & 85.8 & 89 & $\mathbf{+ 3 . 2}$ \\
\hline
\end{tabular}




\section{Conclusions and Future Works}

This paper has addressed a new unsupervised or semi-unsupervised text categorization method. Though our method uses only title words and unlabeled data, it shows reasonably comparable performance in comparison with that of the supervised Naive Bayes classifier. Moreover, it outperforms a clustering method, $\boldsymbol{s I B}$. Labeled data are expensive while unlabeled data are inexpensive and plentiful. Therefore, our method is useful for low-cost text categorization. Furthermore, if some text categorization tasks require high accuracy, our method can be used as an assistant tool for easily creating labeled training data.

Since our method depends on title words and keywords, we need additional studies about the characteristics of candidate words for title words and keywords according to each data set.

\section{Acknowledgement}

This work was supported by grant No. R01-2003000-11588-0 from the basic Research Program of the KOSEF

\section{References}

K. Bennett and A. Demiriz, 1999, Semi-supervised Support Vector Machines, Advances in Neural Information Processing Systems 11, pp. 368-374.

E. Brill, 1995, Transformation-Based Error-driven Learning and Natural Language Processing: A Case Study in Part of Speech Tagging, Computational Linguistics, Vol.21, No. 4.

K. Cho and J. Kim, 1997, Automatic Text Categorization on Hierarchical Category Structure by using ICF (Inverse Category Frequency) Weighting, In Proc. of KISS conference, pp. 507-510.

M. Craven, D. DiPasquo, D. Freitag, A. McCallum, T. Mitchell, K. Nigam, and S. Slattery, 2000, Learning to construct knowledge bases from the World Wide Web, Artificial Intelligence, 118(1-2), pp. 69-113.

T. Joachims, 1998, Text Categorization with Support Vector Machines: Learning with Many Relevant Features. In Proc. of ECML, pp. 137-142.

Y. Karov and S. Edelman, 1998, Similarity-based Word Sense Disambiguation, Computational Linguistics, Vol. 24, No. 1, pp. 41-60.

Y. Ko and J. Seo, 2000, Automatic Text Categorization by Unsupervised Learning, In Proc. of COLING'2000, pp. 453-459.

Y. Ko and J. Seo, 2002, Text Categorization using Feature Projections, In Proc. of COLING'2002, pp. 467-473.
Y. Ko and J. Seo, 2004, Using the Feature Projection Technique based on the Normalized Voting Method for Text Classification, Information Processing and Management, Vol. 40, No. 2, pp. 191-208.

D.D. Lewis, R.E. Schapire, J.P. Callan, and R. Papka, 1996, Training Algorithms for Linear Text Classifiers. In Proc. of SIGIR'96, pp.289-297.

Y. Maarek, D. Berry, and G. Kaiser, 1991, An Information Retrieval Approach for Automatically Construction Software Libraries, IEEE Transaction on Software Engineering, Vol. 17, No. 8, pp. 800813.

A. McCallum and K. Nigam, 1998, A Comparison of Event Models for Naive Bayes Text Classification. AAAI '98 workshop on Learning for Text Categorization, pp. 41-48.

K. P. Nigam, A. McCallum, S. Thrun, and T. Mitchell, 1998, Learning to Classify Text from Labeled and Unlabeled Documents, In Proc. of AAAI-98.

K. P. Nigam, 2001, Using Unlabeled Data to Improve Text Classification, The dissertation for the degree of Doctor of Philosophy.

N. Slonim, N. Friedman, and N. Tishby, 2002, Unsupervised Document Classification using Sequential Information Maximization, In Proc. of SIGIR'02, pp. 129-136.

Y. Yang and J. P. Pedersen. 1997, Feature selection in statistical leaning of text categorization. In Proc. of ICML'97, pp. 412-420.

Y. Yang, S. Slattery, and R. Ghani. 2002, A study of approaches to hypertext categorization, Journal of Intelligent Information Systems, Vol. 18, No. 2. 